\title{
Caracterização da Saúde Mental em Crianças e Adolescentes em Acolhimento Institucional
}

\author{
Jordana Calcing \\ Instituto de Desenvolvimento Educacional do Alto Uruguai \\ Getúlio Vargas, RS, Brasil \\ Silvia Pereira da Cruz Benetti \\ Universidade do Vale do Rio dos Sinos \\ São Leopoldo, RS, Brasil
}

\begin{abstract}
RESUMO
Este estudo teve como objetivo identificar as experiências de vida traumáticas e a prevalência de problemas de comportamento em crianças e adolescentes em situação de acolhimento institucional. O grupo investigado foi constituído por 41 crianças e adolescentes de ambos os sexos, com idade de 7 a 18 anos, provenientes de cinco casas de acolhimento localizadas na região norte do estado do Rio Grande do Sul. Para a obtenção dos dados, utilizaram-se os instrumentos: Inventário de Eventos Estressores na Infância e Adolescência (IEEIA), Inventário de Depressão Infantil (CDI), e o Child Behavior Checklist (CBCL). Os dados apontaram para a presença de problemas de comportamento em um número significativo de crianças e adolescentes, tendo em vista suas histórias de vida marcadas por eventos traumáticos. Considerando esses dados, destaca-se a importância de instituir políticas públicas que contemplem as necessidades de crianças e adolescentes institucionalizados, a fim de que seus direitos sejam garantidos.
\end{abstract}

Palavras-chave: Crianças; Adolescentes; Abrigo; Políticas públicas.

\begin{abstract}
Mental Health Characterization in Institutionalized Children and Adolescents

The objective of this study was to identify traumatic experiences and the prevalence behavioral problems in institutionalized children and adolescents. Forty one children and adolescents between 7 and 18 years old constituted the group investigated from five governmental and non-governmental homes located in north region of Rio Grande do Sul state. To obtain data, the Stressful Life Events Inventory (IEEIA), the Child Depression Inventory (CDI), and the Child Behavior Checklist (CBCL). Data indicated the presence of emotional disorders in a significant number of children, considering life trajectories damaged with traumatic events. Considering these aspects, it is important to develop public politics which address the needs of institutionalized children and adolescents in order that their rights are guaranteed.
\end{abstract}

Keywords: Children; Adolescents; Shelter; Public Policies.

\section{RESUMEN}

\section{Caracterización de la Salud Mental en Niños y Adolescentes Institucionalizados}

Este estudio tuvo como objetivo identificar las experiencias traumáticas de vida y la prevalencia de problemas de conducta en niños y adolescentes en acogimiento residencial. El grupo de estudio consistió en 41 niños y adolescentes de ambos sexos, con edades entre 7-18 años, de cinco refugios ubicados en la región norte del estado de Rio Grande do Sul. Para obtener los datos, se utilizó instrumentos: Inventario de eventos estresantes en la Infancia y la Adolescencia (IEEIA), el Inventario de Depresión Infantil (CDI), Child Behavior Checklist (CBCL). Los datos indicaron la presencia de trastornos emocionales en un número significativo de niños y adolescentes, teniendo en cuenta sus historias de vida marcadas por acontecimientos traumáticos. Teniendo en cuenta estos datos, se destaca la importancia de desenvolver políticas públicas que atiendan las necesidades de los niños y adolescentes institucionalizados, por lo que sus derechos están garantizados.

Palabras clave: Niños; Adolescentes; Refugio; Políticas públicas. 
De acordo com o Estatuto da Criança e do Adolescente (ECA), a retirada da criança do convívio familiar é justificada sempre que seus direitos forem ameaçados ou violados, por ação ou omissão da sociedade ou do Estado, por falta, omissão ou abuso dos pais ou responsável, e em razão de suas condutas. Nestes casos, o acolhimento institucional configura-se como uma medida excepcional e provisória de proteção aos direitos da criança e do adolescente (ECA, 1990).

Estima-se que, atualmente, cerca de $30 \mathrm{mil}$ crianças e adolescentes brasileiros vivam em situação de acolhimento institucional, divididos em 1876 casas governamentais e não governamentais espalhadas por todo o país (Conselho Nacional de Justiça, CNJ, 2011). Entre os riscos e motivos potenciais que levam ao acolhimento institucional no Brasil, encontram-se a negligência, a pobreza, o abandono, a violência e a dependência química dos pais ou responsável (IPEA, 2003; Brasil, 2009). Assim, a determinação de acolhimento institucional sustenta-se em uma intervenção protetora dos direitos da criança e do adolescente, em situação de vulnerabilidade, justamente por visar à garantia desses direitos, principalmente os de proteção e cuidado. Contudo, dados fornecidos pelo Fundo das Nações Unidas para a Infância (UNICEF, 2011), indicam que os fatores que comprometem o desenvolvimento saudável da criança e do adolescente incluem a pobreza e a pobreza extrema; a baixa escolaridade; a exploração do trabalho; a privação da convivência familiar e comunitária; a violência que resulta em assassinatos de adolescentes; a gravidez; a exploração e o abuso sexual; as Doenças Sexualmente Transmissíveis e AIDS; e o abuso de drogas. A privação da convivência familiar e comunitária, associada ao acolhimento institucional, encontra-se em $4^{\circ}$ lugar entre esses nove indicadores. Portanto, ainda que a garantia dos direitos da criança e do adolescente se constitua como meta fundamental da institucionalização, essa experiência deixa marcas importantes no desenvolvimento dessa população.

Em geral, quando encaminhados a casas de acolhimento institucional, crianças e adolescentes trazem consigo uma bagagem de maus-tratos, negligência e vivências anteriores potencialmente traumáticas. O impacto dessas vivências constitui graves riscos para o desenvolvimento, tanto intelectual como emocional, com repercussão nas fases posteriores - a adolescência e a vida adulta (Erol, Simsek, \& Munir, 2010). Assim, crianças expostas ao acolhimento institucional apresentam maiores índices de depressão, dificuldades escolares, sentimento de culpa e desamparo - o que aumenta o risco de envolvimento com drogas e álcool (Johnson, Browne, \& Hamilton-Giachritsis,
2006, Mota, \& Matos, 2008, Schmid, Goldbeck, Nuetzel, \& Fegert, 2008).

Apesar desses aspectos, as casas de acolhimento esforçam-se para oferecer a possibilidade de transformação dessas trajetórias através do estabelecimento de novos vínculos - com os cuidadores e com as demais crianças. Desse modo, esta casa de acolhimento tem a potencialidade de se tornar um espaço de construção de novos relacionamentos afetivos significativos, com dimensões diferentes daqueles experimentados fora dela (Dalben, \& Dell'Aglio, 2008). Ainda assim, considerando a alta prevalência de indicadores clínicos emocionais em crianças e adolescentes cujas trajetórias de vida são marcadas por vivências traumáticas, verifica-se a necessidade de estudos que priorizem a identificação das características emocionais desse grupo social com a finalidade de oferecer subsídios para a atuação dos profissionais da área (Martins, 2005). Portanto, este estudo teve como objetivo principal investigar a prevalência de problemas de comportamento em um grupo de crianças e adolescentes em situação de acolhimento institucional, bem como as experiências de vida traumáticas dessas crianças.

\section{MÉTODO}

Trata-se de um estudo com delineamento quantitativo descritivo, transversal, que procurou identificar as características de saúde mental em crianças e adolescentes institucionalizados, por motivo de afastamento familiar, na região norte do estado do Rio Grande do Sul.

\section{Participantes}

Os participantes foram 41 crianças e adolescentes provenientes de cinco casas de acolhimento governamentais e não governamentais localizadas na região norte do estado do Rio Grande do Sul. As casas governamentais eram subsidiadas pela Prefeitura de dois municípios da região norte, acolhendo, em média, 15 crianças. Por sua vez, as casas não governamentais eram financiadas por ONGs e funcionavam também como lares de acolhimento. Em termos de organização e estrutura de funcionamento todas as casas tinham boa infraestrutura física e humana.

A composição da amostra deu-se a partir de uma listagem, fornecida pelo(a) Coordenador(a) de cada casa de acolhimento. A seleção dos participantes foi feita de forma não aleatória, adotando como critérios de inclusão: a faixa etária (7 a 18 anos), o tempo de institucionalização (mínimo de seis meses) e a ausência de deficiências que impedissem a aplicação 
dos instrumentos. Assim, de um grupo em torno de 60 crianças e adolescentes em acolhimento institucional no momento da coleta de dados, foram selecionados 41 participantes.

\section{Procedimentos}

Mediante a aprovação do Comitê de Ética da Unisinos, CEP 11/055, foram contatados os responsáveis pelas instituições, com o intuito de explicar os objetivos da pesquisa e obter aprovação do trabalho. Após essa etapa inicial, o Termo de Consentimento Livre e Esclarecido (TCLE) foi assinado pelos monitores, pelas crianças e pelos adolescentes. A seguir, foram realizadas visitas de ambientação, quando a pesquisadora pôde conhecer o espaço físico das casas, os profissionais e os sujeitos da pesquisa. A aplicação dos instrumentos ocorreu conforme agendamento de horários com os coordenadores de cada casa e com as crianças. A coleta transcorreu durante quatro meses, sendo em média entrevistados cinco sujeitos por semana. Os instrumentos foram aplicados de maneira coletiva e individual, de acordo com as demandas de cada criança, levando em conta a sua faixa etária e a sua capacidade intelectual. Mesmo os participantes que responderam de forma coletiva aos questionários contavam com o auxílio de uma ou duas pesquisadoras, prontas para atendê-los, caso necessitassem de ajuda.

\section{Instrumentos}

Ficha de Dados Sociodemográficos. Visando a identificar as principais características pessoais e familiares das crianças e dos adolescentes institucionalizados, foi aplicado um questionário de dados sociodemográficos aos monitores das casas, elaborado com base no instrumento desenvolvido por Serafini (2004). O questionário inclui questões sobre idade, filiação, número de irmãos, motivos do acolhimento, contato com os familiares, além de questões sobre o comportamento e o relacionamento dentro da instituição com os colegas e com os próprios monitores.

Child Behavior Checklist (CBCL. Para investigar manifestações clínicas na infância e adolescência, foi utilizado o Child Behavior Checklist (CBCL), que é um Inventário de Comportamentos na Infância e Adolescência (Achenbach, 1991). No Brasil, o CBCL foi validado por Bordin, Mari e Caeiro (1995). A primeira parte avalia a Competência Social e é constituída por três escalas individuais Atividades, Sociabilidade e Escolaridade. A segunda parte avalia Problemas de Comportamento e possui oito escalas de síndromes - Retraimento, Queixas Somáticas, Ansiedade/Depressão, Problemas Sociais, Problemas do Pensamento, Problemas de
Atenção, Problemas Sexuais, Comportamento de Quebrar Regras e Comportamento Agressivo. As três primeiras subescalas correspondem à escala de comportamentos do tipo internalização, enquanto as duas últimas subescalas correspondem à escala de comportamentos do tipo externalização. A soma das escalas permite a classificação dos valores através de escores padronizados: categoria não clínica: abaixo de 63 pontos; categoria limítrofe: 64 pontos; e categoria clínica: acima de 65 pontos.

Inventário de Eventos Estressores na Infância e Adolescência (IEEIA). Este instrumento é composto por 64 itens na forma de eventos de vida estressores. Para cada item, o participante deve indicar, em uma alternativa sim/não, se o evento ocorreu e, a partir disso, em uma escala Likert de cinco pontos, informar o impacto atribuído a cada evento. Essa versão adaptada apresentou elevada consistência interna (alpha de Cronbach $=0,92$ ), mostrando-se confiável na avaliação de eventos de vida estressores em adolescentes. Foram avaliados os escores referentes ao número total de eventos (ET), à média de eventos (ME) e à intensidade de eventos (IE) (Kristensen, Leon, D'Incao, \& Dell'Aglio, 2004).

Inventário de Depressão Infantil (CDI). O inventário foi validado para o Brasil em 1995, por Gouveia, Barbosa, Almeida e Gaião (1995). O CDI é um instrumento que avalia sintomas afetivos, cognitivos e comportamentais na depressão infantil e adolescente. É composto de 27 itens, cada um dos quais possui três alternativas de resposta cujos valores variam de 0 a 2 pontos. Dessas três alternativas, uma corresponde à normalidade, outra à severidade dos sintomas e outra à enfermidade clínica mais significativa. O respondente é orientado a selecionar a alternativa que melhor descreva seus sentimentos nas últimas semanas (Cruvinel, Boruchovitch, \& Santos, 2008). A análise fatorial do CDI em jovens brasileiros (Wathier, Dell'Aglio, \& Bandeira, 2008) indicou que indivíduos que atingem valores acima do percentil 85 apresentam características clínicas de depressão. Para meninos, tanto na faixa etária de 7-12 quanto na de 1317 anos de idade, o ponto de corte foi de 14; para as meninas na faixa etária de 7-12 anos, o valor foi de 16; já na faixa etária entre 13-17 anos de idade foi de 18 .

\section{Análise dos dados}

Os dados foram analisados através de testes estatísticos descritivos (frequências e médias), testes inferenciais paramétricos (Análise de Variância e Correlação de Pearson) e testes não paramétricos (Qui-quadrado) através do Programa Estatístico SPSS, versão 19. 


\section{Considerações éticas}

Após a aprovação pelo Comitê de Ética da UNISINOS (Projeto $n^{\circ}$ CEP 11/055), foi obtido o Consentimento Informado da Instituição que mantém formalmente a guarda das crianças e dos adolescentes institucionalizados, bem como dos monitores que participaram da coleta de dados, conforme orientações éticas para pesquisas com seres humanos.

\section{RESULTADOS}

Os participantes do presente estudo foram 41 crianças e adolescentes, sendo $24(58,5 \%)$ do sexo masculino. Desse grupo, 22 participantes tinham entre 7 e 12 anos de idade $(53,7 \%)$ e $19(46,3 \%)$ entre 12 e 18 anos de idade. A média de idade do sexo masculino foi de $\mathrm{M}=11,50(\mathrm{DP}=3,23)$ e do feminino, $\mathrm{M}=11,52$ $(\mathrm{DP}=3,60)$.

O contexto institucional foi descrito pelas crianças e pelos adolescentes como satisfatório: $78 \%$ dos participantes mencionaram que o relacionamento com os cuidadores e com as outras crianças era bom. Todo o grupo estava envolvido em atividades na instituição, as quais incluíam participação em tarefas de cuidado da casa, jogos, televisão, videogame, passeios, brinquedos em geral e atividades escolares.

Com relação à escolaridade, constatou-se que 92,7\% das crianças e dos adolescentes estavam frequentando a escola no momento da coleta. Entretanto, grande parte $(46,3 \%)$ encontrava-se entre o $1^{\circ}$ e o $3^{\circ}$ ano do ensino fundamental. A análise das idades em relação às séries correspondentes indicou que o grupo entre a $4^{\mathrm{a}}$ e a $6^{\mathrm{a}}$ série apresentou maior atraso em relação à escolaridade. A maior frequência de repetência escolar ocorreu entre os meninos $\left(2 \times 2, \chi^{2}=5,427, p .02\right)$.

Observando-se os dados da Tabela 1 verifica-se que $61 \%$ das crianças mantinham contato com algum familiar. Os participantes que estavam acolhidos há menos de dois anos, 19 (79,2\%) mantinham contato com os pais, enquanto do grupo com mais de dois anos de acolhimento somente nove $(33,3 \%)\left(2 \times 2, \chi^{2}=8.19\right.$, $p<.00)$. Além disso, diversas crianças dividiam a casa de acolhimento com irmãos $(63,4 \%)$. Quanto aos motivos do acolhimento institucional observados no grupo investigado, verificou-se a negligência $(63,4 \%)$, o abandono (9,8\%), o abuso sexual (7,3\%), o abuso físico $(7,3 \%)$ e a doença mental dos pais $(4,9 \%)$ como as causas de institucionalização mais frequentes.

A análise dos dados relativas ao objetivo de identificar os problemas de comportamento no grupo estudado indicou que 26 dos participantes $(63,4 \%)$ obtiveram escores associados à categoria classificada
TABELA 1

Características sociodemográficas e do acolhimento institucional da amostra

\begin{tabular}{|c|c|c|c|}
\hline \multicolumn{2}{|c|}{ Dados Sociodemográficos } & $N$ & $\%$ \\
\hline \multirow[t]{4}{*}{ Idade } & $7-9$ anos & 15 & 36,6 \\
\hline & 10-12 anos & 12 & 29,3 \\
\hline & 13-15 anos & 5 & 12,2 \\
\hline & $>16$ anos & 9 & 22,0 \\
\hline \multirow[t]{2}{*}{ Frequenta a escola } & Sim & 39 & 95,1 \\
\hline & Não & 2 & 4,9 \\
\hline \multirow{3}{*}{$\begin{array}{l}\text { Números de irmãos } \\
\text { na instituição }\end{array}$} & Até 3 & 17 & 41,4 \\
\hline & Mais de 3 & 22 & 53,6 \\
\hline & Nenhum & 2 & 5,0 \\
\hline \multirow[t]{2}{*}{ Contato com os pais } & Sim & 25 & 61,0 \\
\hline & Não & 16 & 39,0 \\
\hline \multirow[t]{2}{*}{ Fuga da instituição } & Sim & 8 & 19,5 \\
\hline & Não & 33 & 80,5 \\
\hline \multirow{6}{*}{$\begin{array}{l}\text { Tempo de } \\
\text { institucionalização }\end{array}$} & 1 a 6 meses & 11 & 26,8 \\
\hline & 6 meses a 1 ano & 6 & 14,6 \\
\hline & 1 a 2 anos & 7 & 17,1 \\
\hline & 2 a 3 anos & 6 & 14,6 \\
\hline & Mais de 3 anos & 9 & 22,0 \\
\hline & Sem informação & 2 & 4,9 \\
\hline \multirow[t]{3}{*}{ Série escolar } & $1^{\mathrm{a}}$ a $3^{\mathrm{a}}$ série & 19 & 46,3 \\
\hline & $4^{\circ}$ a $6^{a}$ série & 17 & 41,5 \\
\hline & $7^{a}$ série em diante & 3 & 7,3 \\
\hline \multirow[t]{6}{*}{ Motivo do acolhimento } & Negligência & 26 & 63,4 \\
\hline & Abandono & 4 & 9,8 \\
\hline & Abuso físico & 3 & 7,3 \\
\hline & Morte dos pais & 3 & 7,3 \\
\hline & Abuso sexual & 3 & 7,3 \\
\hline & Doença mental dos pais & 2 & 4,9 \\
\hline
\end{tabular}

como clínica, segundo a avaliação do CBCL. No grupo, o sexo masculino apresentou uma frequência significativamente maior de diagnóstico clínico $(\mathrm{N}=75 \%)(2 \times 2,9.862, p .00)$. Dentre as síndromes internalizantes (retraimento, ansiedade e somatização) e externalizantes (agressividade e quebrar regras), além da dificuldade de atenção, foram observadas as seguintes características, conforme a Tabela 2, adiante.

Em relação à faixa etária, o grupo de adolescentes apresentou um maior número de casos clínicos $(\mathrm{N}=10,52,6 \%)$ do que as crianças $(\mathrm{N}=2,9,1 \%)(3 \times 2$, $\left.\chi^{2}=10.52, p .00\right)$. As síndromes de ansiedade (41,5\%), quebrar regras $(41,5 \%)$ e dificuldade de atenção $(36,6 \%)$ foram os três quadros clínicos mais frequentes. Não houve diferenças de diagnóstico entre os sexos em nenhuma faixa etária. Entretanto, agressividade e dificuldade de atenção foram mais frequentes entre o sexo masculino na faixa etária entre 7 e 12 anos. $O$ teste de correlação de Pearson entre as variáveis relativas aos escores internalizantes e externalizantes não apontou associação significativa de manifestação de escores clínicos com o tempo de permanência das crianças na instituição e com contato com os familiares. 
TABELA 2

Características diagnósticas no grupo de crianças e adolescentes

\begin{tabular}{lccccc}
\hline \multirow{2}{*}{$\begin{array}{c}\text { Sindromes } \\
\text { CBCL }\end{array}$} & \multicolumn{2}{c}{ Crianças } & \multicolumn{2}{c}{ Adolescentes } & Total \\
\cline { 2 - 6 } & $N=12$ & $N=10$ & $N=12$ & $N=7$ & $N=41$ \\
\hline Internalização & & & & & \\
Retraimento & $3(25 \%)$ & $3(30 \%)$ & $4(33,3 \%)$ & 0 & $10(24,4 \%)$ \\
Ansiedade & $6(50 \%)$ & $2(20 \%)$ & $6(50 \%)$ & $3(42,9 \%)$ & $17(41,5 \%)$ \\
Somatização & $1(8,3 \%)$ & $1(11 \%)$ & $3(25 \%)$ & $2(28,6 \%)$ & $7(17,1 \%)$ \\
Atenção & $8(66,7 \%)^{*}$ & $2(20 \%)$ & $3(25 \%)$ & $2(28,6 \%)$ & $15(36,6 \%)$ \\
Externalização & & & & & \\
Agressividade & $6(50 \%)^{* *}$ & $1(8,35)$ & $1(10 \%)$ & $1(14,3 \%)$ & $9(22 \%)$ \\
Quebrar regras & $8(66,7 \%)$ & $3(30 \%)$ & $5(41,7 \%)$ & $1(14,3 \%)$ & $17(41,5 \%)$ \\
\hline
\end{tabular}

$* 2 \times 2, \chi^{2}=4.19, p=.05 ; * * 2 \times 2, \chi^{2}=5.04, p=.03$.

Para obter-se uma análise mais específica de aspectos de saúde mental, a frequência dos sintomas depressivos foi avaliada através do inventário CDI. Em relação ao diagnóstico depressivo, verificou-se um total de 22 crianças/adolescentes $(53,7 \%)$ classificados como sem sintomas depressivos e 19 como com sintomas na área clínica $(46,3 \%)$. A média total no CDI foi de $\mathrm{M}=13,20$ e $\mathrm{DP}=8,13$, não havendo diferenças significativas entre os sexos. Especificamente no grupo infantil, a média foi de $\mathrm{M}=12,50(\mathrm{DP}=6,20)$; no grupo de adolescentes, a média foi de $M=14,06(D P=10,13)$. Tal diferença não considerada significativa. Em termos classificatórios, no grupo de crianças, 11 casos (50\%) obtiveram escores clínicos, e no grupo de adolescentes, oito casos (42,1\%). Não houve diferenças entre os sexos quanto à manifestação de diagnóstico depressivo.

Eventos estressores. Em relação à média dos eventos e à intensidade percebida, não houve diferenças significativas entre o sexo masculino e o feminino. Analisando-se a média dos eventos, não houve diferenças entre o sexo masculino $(\mathrm{M}=27,13, \mathrm{DP}=8,43)$ e o feminino $(M=26,24, D P=9,84)$, sendo que para ambos os sexos o evento estressor mais mencionado foi a entrada na casa de acolhimento. No que diz respeito à intensidade dos eventos, não houve diferença significativa entre os sexos (Masculino $=\mathrm{M}=89,58$, $\mathrm{DP}=39,61$, Feminino $=98,71(44,59)$. Para o masculino, o evento sentido como mais intenso é Ser estuprado $(\mathrm{M}=5,00, \mathrm{DP}=.00)$, seguido de Ter sofrido violência, Ter dormido na rua $(\mathrm{M}=4,40,1,34)$, Morte de irmãos $(\mathrm{M}=4,40, \mathrm{DP}=1,34)$. Para o feminino, Sentir-se rejeitado(a) por colegas e amigos(as) $(\mathrm{M}=5,00, \mathrm{DP}=.00)$, Separação dos pais $(\mathrm{M}=5,00$, $\mathrm{DP}=.00)$, Ser rejeitado pelos familiares $(\mathrm{M}=5,00$, $\mathrm{DP}=.00)$. Observa-se uma diferença significativa entre o sexo masculino e feminino no que diz respeito aos eventos de vida citados. Meninas destacam como estressantes as situações ligadas às relações de pares, enquanto que para os meninos as experiências mais traumáticas estão associadas à violência, agressão e perdas.

Analisando-se a frequência dos eventos em relação às faixas etárias de crianças (7-12) e adolescentes (13-18), observou-se que Ter que viver no abrigo foi o evento mais citado em ambos os grupos. Há diferenças, entretanto, no sentido de que situações de estresse envolvendo familiares (Ser impedido de ver os pais, $81,8 \%$ ) e irmãos (Brigas com irmãos, $77,3 \%$ ) foram citadas com mais frequência pelas crianças, enquanto, entre os jovens, Mudança de escola $(94,7 \%)$ foi o evento identificado pela maioria como estressante. Ainda assim, Brigas com irmãos (84,2\%), Tirar notas baixas na escola $(78,9 \%)$ e Separação dos pais $(73,7 \%)$ apareceram também como muito frequentes.

Associação entre manifestações clínicas e características dos eventos estressores. O teste ANOVA não apontou diferença significativa entre os grupos de crianças e adolescentes com ou sem diagnóstico clínico em relação ao total de eventos vivenciados. Entretanto, a análise dos diagnósticos específicos, internalizantes e externalizantes apontou que meninas com diagnóstico clínico de externalização apresentaram uma média significativamente maior na intensidade percebida dos eventos $(M=128, D P=49,03)$ se comparada com a dos meninos $(M=79, D P=41,07)$. Considerando os resultados do CDI, percebe-se que houve uma correlação significativa entre o escore depressivo e a intensidade dos eventos $(r .376, p .015)$, indicando que, quanto maior o escore de depressão, mais intensos eram percebidos os eventos traumáticos para ambos os sexos. 


\section{DISCUSSÃO}

A preocupação em identificar e conhecer as especificidades das demandas de crianças e adolescentes acolhidos institucionalmente, no tocante aos aspectos da saúde mental dessa população, reflete a necessidade de oferecer um atendimento institucional que responda às vulnerabilidades inerentes ao processo de institucionalização. Tanto a literatura nacional (Nogueira, 2004, Parreira, \& Justo, 2005, Cavalcante, Magalhães, Pontes, \& 2007, Brasil, 2009) como a internacional (Smyke, Dumitrescu, \& Zeanah, 2002, Johnson, Browne, \& Hamilton-Giachritsis, 2006, Erol, Simsek, \& Munir, 2010) apontam para os maiores riscos dessas crianças e jovens desenvolverem transtornos emocionais relativos às vivências traumáticas ocorridas ao longo de suas trajetórias de vida. No entanto, reconhecer esses aspectos não significa assumir a perspectiva de que essas trajetórias não possam ser revertidas e narradas sob a perspectiva da resiliência, conceito que se refere ao processo de superação ou administração dos efeitos negativos da exposição aos eventos traumáticos, resultando em desenvolvimento adaptado apesar das adversidades (Yunes, 2003, Siqueira, \& Dell'Aglio, 2010, Dalbem, \& Dell'Aglio, 2008, Rygaard, 2008).

Sustentando-se na perspectiva de conhecer para poder atuar e intervir, este trabalho teve justamente o interesse de identificar as características de saúde mental de crianças e adolescentes institucionalizados, bem como as experiências traumáticas ocorridas ao longo do seu desenvolvimento. Inicialmente, considerando a experiência de institucionalização, todo o grupo investigado provinha de casas-lares administradas pelos municípios ou por ONGs as quais ofereciam às crianças a possibilidade de realizar diversas atividades sociais, educacionais e de lazer. Além disso, a maioria frequentava a escola, fato que se constitui em um importante elemento socializador, além de educativo e promotor de saúde mental.

No entanto, ainda que o aspecto da manutenção de vínculos escolares tenha sido prevalente, foi observado um importante atraso escolar em relação às séries correspondentes às faixas etárias, que pode estar associado a dificuldades de aprendizagem, ou mesmo a um possível afastamento da escola em função dos problemas enfrentados antes ou durante o acolhimento. A esfera escolar, portanto, constitui um dos primeiros aspectos a serem indicados como merecedores de uma atenção detalhada por parte dos profissionais envolvidos com o cuidado desses grupos, tanto pela questão educativa em si, como pelo papel importante que a escola assume na vida dessas crianças e adolescentes, por ser um ambiente fundamental de socialização e integração comunitária.

Em geral, os estudos apontam a negligência de cuidados como um dos principais motivos que levam ao acolhimento institucional. Da população pesquisada no presente estudo, $63,4 \%$ foi encaminhada ao cuidado institucional por negligência. Esse dado corrobora os estudos realizados por Cavalcante, Magalhães e Pontes (2007) e pelo Ministério da Saúde (Brasil, 2009), segundo os quais a maioria dos casos de institucionalização ocorre devido à negligência da família. Entretanto, o relatório da Organização Mundial da Saúde sobre saúde e violência (Krug et al., 2002) menciona que a negligência diz respeito às falhas dos pais em proporcionar condições para o crescimento da criança, em uma ou mais das seguintes áreas: saúde, educação, desenvolvimento emocional, nutrição, abrigo e condições de vida seguras. Todavia, o relatório destaca que a negligência distingue-se da situação de pobreza de uma população, pelo fato de que, no primeiro caso, as famílias têm a seu dispor recursos diretos ou indiretos para oferecer as condições de desenvolvimento à criança, mas isso não é feito. Novamente, aqui, observam-se as questões econômicas e sociais brasileiras que fragilizam a capacidade de manutenção dos laços familiares e colocam crianças e adolescentes em contextos de grande vulnerabilidade, com graves consequências para seu desenvolvimento em geral. Nesses casos, a institucionalização vem refletir uma necessidade social mais ampla de atenção a um setor da população que vive em precária situação econômica.

Entretanto, é necessário destacar que a negligência também se associa a outros fatores, diferentes da carência de recursos financeiros para manter crianças e adolescentes em condições dignas: há casos de negligência que ocorrem devido ao abuso de álcool e drogas por parte dos pais ou responsáveis, situação que também torna esses grupos negligenciados (FernándezMillán, Hamido-Mohamed, \& Ortiz-Gómez, 2009, Brasil, 2009).

Embora esteja previsto no ECA (1990) que o acolhimento institucional seja uma medida excepcional e provisória, que não deve ter duração maior de dois anos, nesse estudo observou-se que, em 15 casos, o tempo de acolhimento era superior ao previsto. Essa característica indica a dificuldade de reinserção das crianças e dos adolescentes em seu grupo familiar, bem como, naqueles casos de perda de pátrio poder, para a dificuldade de adoção de crianças maiores. Por outro lado, 26 crianças $(63,4 \%)$ tinham irmãos também acolhidos nas instituições, o que indica o esforço de preservar, conforme o ECA, mesmo nos casos de institucionalização, os vínculos familiares. 
Gonçalves (2008) comenta, porém, que, mesmo permanecendo na companhia de irmãos, o acolhimento institucional configura-se como uma medida percebida pelas crianças e pelos adolescentes como estressante. No presente estudo, a questão de ter de viver em abrigo aparece como a principal experiência estressante para os participantes em geral, indicando que, apesar do fato de o acolhimento refletir a extrema vulnerabilidade a que estavam expostos anteriormente no ambiente familiar, a ida para a instituição é vivenciada com sofrimento. Esse dado assinala, como um marco fundamental no trabalho com as crianças e os adolescentes que necessitam ser encaminhados para acolhimento institucional, a importância de um trabalho específico que inclua tanto o momento de institucionalização como o período adaptativo à instituição. Nesse sentido, o rompimento vivido pela criança não se restringe aos laços familiares, mas inclui toda uma identidade, que é rompida e descontinuada a partir do acolhimento. Portanto, a acolhida afetuosa da criança, o apoio constante de técnicos especializados, incluindo o reconhecimento das experiências vividas por ela e, fundamentalmente, o desenvolvimento de estratégias de acompanhamento pedagógico, psicológico e familiar, tem sido apontado como base para uma experiência positiva (Rygaard, 2008).

Além do aspecto acima, foi identificada, entre os participantes deste estudo, uma média de ocorrência de $27,13(\mathrm{DP}=8,43)$ eventos entre o sexo masculino e de $26,24(\mathrm{DP}=9,85)$ entre o feminino. Da mesma forma, respectivamente, a intensidade média percebida dos eventos foi de $89,58(39,62)$ para o masculino e 98,71 $(44,59)$ para o feminino.

$\mathrm{Na}$ investigação de Kristensen, Leon, D'Incao e Dell'Aglio (2005) com 330 adolescentes da região metropolitana da cidade de Porto Alegre, utilizandose igualmente do instrumento IEEIA, foi identificada a média de 17,03 eventos $(\mathrm{DP}=9,4)$ entre os participantes, e impacto de 50,02 (DP=37,83). Da mesma forma, Benetti, Pizetta, Schwartz, Hass e Melo (2010), em um grupo de 245 adolescentes da região metropolitana de Porto Alegre, verificaram uma média geral de ocorrência dos eventos estressores similar para ambos os sexos: feminino $(\mathrm{M}=17,00, \mathrm{DP}=10,05) \mathrm{e}$ masculino $(\mathrm{M}=15,83, \mathrm{DP}=9,87)$. Ao contrário, no estudo de Poletto, Koller e Dell'Aglio (2009), 142 participantes residiam com suas famílias e 155 eram institucionalizados. As crianças institucionalizadas tiveram uma média de eventos estressores de $\mathrm{M}=26,79(\mathrm{SD}=8,67)$, enquanto, entre as crianças que residiam com suas famílias, a média foi de $\mathrm{M}=19,16$ $(\mathrm{SD}=9,37)$. Já em um estudo longitudinal (WathierAbaid, Dell'Aglio, \& Koller, 2010), 127 crianças e adolescentes entre 7 e 16 anos de idade, provenientes de sete instituições de proteção governamentais e duas não-governamentais, na região metropolitana de Porto Alegre, foram avaliados em dois momentos quanto à ocorrência de eventos de vida estressantes, ao número de irmãos, à configuração familiar, ao tempo de institucionalização e à presença de depressão. Também foram investigados os preditores de sintomas depressivos no grupo avaliado. Nesse estudo, a média dos eventos estressores na primeira aplicação do IEEIA foi de 26,57 $(\mathrm{SD}=8,56)$ e no segundo momento, de $25,91(\mathrm{SD}=9,33)$. Nos dois momentos, cerca de 40 crianças apresentavam escore compatível com o diagnóstico de depressão.

Com base nesses resultados, verifica-se que distintamente do que ocorre com crianças e adolescentes não institucionalizados, as experiências de vida de crianças acolhidas é marcada por vivências traumática. Assim, as crianças mencionaram o castigo, o impedimento de ver os pais e as brigas com irmãos como os eventos mais frequentes. Já os adolescentes citaram a mudança de escola, ir para o conselho tutelar, brigas com irmãos, tirar notas baixas na escola. Por conseguinte, nas crianças, a separação familiar e o resultante impedimento de convívio caracteriza maior dependência afetiva, que, nos adolescentes, é traduzida pelo estresse de trocas de escola, fato que rompe com alianças e amizades entre o grupo de pares. Chama a atenção, porém, o aspecto positivo da identificação pelos adolescentes das dificuldades escolares como uma situação negativa, visto que demonstra o reconhecimento do processo educativo como relevante em seu contexto.

Em uma pesquisa realizada na Alemanha, que envolveu a investigação de 689 crianças e adolescentes de 4 a 18 anos de idade, que viviam em casas de acolhimento institucional (Schmid et al., 2008), foi verificado um elevado número de crianças com transtornos mentais e de conduta: 59\% preenchiam os critérios diagnósticos para depressão. Esse estudo apontou a alta prevalência de comportamentos externalizantes e disruptivos, principalmente entre o sexo masculino, enquanto o feminino manifestava maior prevalência de transtornos internalizantes. Da mesma forma, no presente estudo, constatou-se que 26 dos participantes apresentavam escores compatíveis com a classificação clínica segundo o CBCL, sendo que a maior frequência de casos atingia o sexo masculino. Uma análise mais detalhada das distintas síndromes que compõem os quadros clínicos externalizantes e o internalizante verificou, entre os meninos, uma maior manifestação de comportamentos agressivos e de dificuldades de atenção. 
A característica de maior prevalência de diagnóstico depressivo entre as meninas não foi detectada no presente trabalho, fato que talvez se deva ao tamanho da amostra. Ainda assim, entre as meninas com diagnóstico clínico de externalização, observouse uma importante característica em relação a maior intensidade percebida dos eventos, se comparada a dos meninos. Da mesma forma, a intensidade dos eventos para ambos os sexos associou-se ao diagnóstico de depressão. Esses dados apontam para a necessidade de intervenções imediatas face às vivências traumáticas e também o acompanhamento de crianças e adolescentes. Porém, é essencial a criteriosa identificação de como a própria criança percebeu aquele evento traumático para poder ser desenvolvido um programa adequado de intervenção.

\section{CONSIDERAÇÕES FINAIS}

Com o objetivo de identificar as experiências de vida traumáticas e a prevalência de transtornos emocionais em crianças e adolescentes em situação de acolhimento institucional, este estudo indicou a importância do desenvolvimento de intervenções em saúde mental dirigidas a essa população. Tomados em conjunto, os dados apontaram para a presença de transtornos emocionais em um número significativo de crianças e adolescentes, que relataram histórias de vida marcadas por eventos traumáticos e, sobretudo, vivenciaram o acolhimento como uma situação estressante.

Ainda que as instituições atuem de forma positiva, ao oferecerem um espaço organizado, com horários e atividades definidas, proporcionando uma situação diversa da oferecida pelo ambiente anterior de privação, é evidente que as necessidades emocionais das crianças e adolescentes necessitam de uma atenção especializada. Por esta razão, torna-se indispensável que a equipe técnica esteja preparada para acolher cada criança e adolescente em sua entrada na casa, realizando um trabalho dirigido especificamente para as questões de prevenção e intervenção em saúde mental. Pelos dados verificados neste trabalho, as manifestações de sofrimento psíquico associadas às trajetórias de vida de perdas e violência demandam dos profissionais um atendimento altamente especializado, tanto para o reconhecimento das necessidades de cada criança como para o desenvolvimento de planejamento da intervenção psicossocial que será realizada em cada caso.

Dessa forma, como conclusão deste trabalho, destacamos o trabalho de resgate de vínculos na dimensão familiar, o investimento criterioso de apoio ao processo educativo das crianças, o treinamento dos técnicos e, principalmente, o atendimento das demandas emocionais das crianças e adolescentes como medidas para o atendimento nas situações de acolhimento institucional. Somente intervindo nesses contextos de forma integrada à rede de atendimento estadual e municipal, estaremos transformando trajetórias marcadas por privação e sofrimento.

\section{REFERÊNCIAS}

Achenbach, T. M. (1991). Integrative Guide for the CBCL/4-18, YSR, and TRF Profiles, University of Vermont, department of Psychiatry, Burlington, VT, pp. 1-211. doi: 10.1037/0021843X.106.3.425

Baptista, M. V. (2006). Abrigo: comunidade de acolhida e socioeducação. Em M. V. Baptista (Org.), Um olhar para a história (pp. 24-37). São Paulo: Instituto Camargo Corrêa.

Benetti, S. P. C., Pizetta, A., Schwartz, C. B., Hass, R. A., \& Melo, V. L. (2010). Problemas de saúde mental na adolescência: características familiares, eventos traumáticos e violência. PsicoUSF, 15, 321-332. Recuperado de http://www.scielo.br/ scielo. php?script=sci_arttext\&pid=S1413-82712010000300006\&.

Bordin, I. A. S., Mari, J. J., \& Caeiro, M. F. (1995). Validação da versão brasileira do Child Behavior Checklist (CBCL) (Inventário de Comportamentos da Infância e Adolescência): Dados Preliminares. Revista da ABP-APAL, 17, 55-66.

Brasil. (2009). Estatuto da Criança e do Adolescente (1990). Lei $\mathrm{n}^{\circ} 8069$, de 13 de julho de 1990. Brasília, DF.

Brasil. Ministério da Saúde (2009). O impacto da violência na saúde das crianças e adolescentes. Brasília, DF.

Cavalcante, L. I. C., Magalhães, C. M. C., \& Pontes, F. A. R. (2007). Institucionalização precoce e prolongada de crianças: discutindo aspectos decisivos para o desenvolvimento. Aletheia, 25, 20-34.

Cruvinel, M., Boruchovitch, E., \& Santos, A. A. A. (2008). Inventário de Depressão Infantil (CDI): análise dos parâmetros psicométricos. Fractal, Revista de Psicologia, 20(2), 473-489. doi: 10.1590/S1984-02922008000200013.

Conselho Nacional de Justiça, Brasil. Recuperado de $<$ http://www. direitolegal.org/noticias-gerais/brasil-tem-mais-de-30-milcriancas-e-adolescentes-vivendo-em-abrigos/>.

Dalbem, J. X. \& Dell'Aglio, D. D. (2008). Apego em adolescentes institucionalizadas: processos de resiliência na formação de novos vínculos afetivos. Psico, 39(1), 33-40.

Erol, N., Simsek, Z., \& Munir, K. (2010). Mental health of adolescents reared in institutional care in Turkey: challenges and hope in the twenty-first century. European Child and Adolescent Psychiatry, 19, 113-124. doi: 10.1007/s00787-009-0047-2

Fernández Millán, J., Hamido-Mohamed, A., \& Ortiz-Gómez, M. M. (2009). Influencia del acogimiento residencial en los menores en desamparo. Education \& Psycology, 7(2), 715-728.

Gonçalves, L. P. M. C. (2008). O impacto da vida em instituições: narrativas e significados em crianças vitimas de maus tratos. Monografia, Faculdade de Ciências Humanas e Sociais, Universidade Fernando Pessoa, Porto.

Gouveia, V. V., Barbosa, G. A., Almeida, H. J. F., \& Gaião, A. A. (1995). Inventário de Depressão Infantil - CDI: Estudo de adaptação com escolares de João Pessoa. Jornal Brasileiro de Psiquiatria, 44, 345-349.

Instituto de Pesquisa Econômica Aplicada (2003). Levantamento Nacional dos Abrigos para Crianças e Adolescentes da Rede de Serviço de Ação Continuada (SAC). Brasília, DF. 
Johnson, R., Browne, K., \& Hamilton-Giachritsis, C. (2006). Young children in institutional care at risk of harm. Trauma, Violence, \& Abuse, 7, 34-60. doi: 10.1177/1524838005283696

Kristensen, C., Leon, J., D’Incao, D., \& Dell'Aglio, D. (2004). Análise da frequência e do impacto de eventos estressores em uma amostra de adolescentes. Interação em Psicologia, 8, 45-55.

Krug, E. G., et al. (2002). World report on violence and health. Geneva: World Health Organization. doi:10.1016/S01406736(02)11133-0

Martins, P. (2005). O acolhimento familiar como resposta de proteção à criança sem suporte familiar adequado. Infância e Juventude, 4, 63-84.

Maclean, K. (2003). The impact of institutionalization on child development. Development and Psychopathology, 15(4). Recuperado de http://journals.cambridge.org/action/displayA bstract? fromPage $=$ online \&aid $=187219$. doi: $10.1037 / 2157$ 3883.1.S.3

Mota, C. P. \& Matos, P. M. (2008). Adolescência e institucionalização numa perspectiva de vinculação. Psicologia e Sociedade, 20(3), 367-377. doi: 10.1590/S0102-71822008000300007

Nogueira, P. C. (2004). A criança em situação de abrigamento: Reparação ou re-abandono. Dissertação de Mestrado, Programa de Pós-Graduação em Psicologia Clínica, Universidade de Brasília, Brasília, Distrito Federal.

Parreira, S. M. C. P. \& Justo, J. S. (2005). A criança abrigada: Considerações acerca do sentido da filiação. Revista Psicologia e Estudo, 10, 175-180.

Poletto, M., Koller S. H., \& Dell'Aglio, D. D. (2009). Eventos estressores em crianças e adolescentes em situação de vulnerabilidade social de Porto Alegre. Ciência \& Saúde Coletiva, 14, 455-466.

Quintãns, C. R. P. (2009). Era uma vez a Instituição onde eu cresci: narrativas de adultos sobre experiências de institucionalização. Dissertação de Mestrado, Instituto de Educação e Psicologia, Universidade do Minho, Portugal.

Ramos, M. E. C. \& Oliveira, K. D. (2008). A transmissão geracional em diferentes contextos. In M.A. Penso \& L.F. Costa (Org.). Transgeracionalidade percebida nos casos de maus-tratos (pp. 99-122). São Paulo: Summus.

Rygaard, N. P. (2008). El nino abandonado: Guia para el tratamiento de los transtornos del apego. Barcelona: Gedisa.

Schmid, M., Goldbeck, L., Nuetzel, J., \& Fegert, J. M. (2008). Prevalence of mental disorders among adolescents in German youth welfare institutions. Child and Adolescent Psychiatry Mental Health, 28, 2-2. doi: 10.1186/1753-2000-2-2.
Serafini, A. J. (2004). Teste das Fábulas: um estudo com crianças abrigadas. Dissertação de Mestrado, Instituto de Psicologia, Universidade Federal do Rio Grande do Sul, Porto Alegre.

Siqueira, A. C. \& Dell'Aglio, D. D. (2010). Crianças e adolescentes institucionalizados: desempenho escolar, satisfação de vida e rede de apoio social. Psicologia: Teoria e Pesquisa, 26(3), 407-415.

Smyke, A. T., Dumitrescu, A., \& Zeanah, C. H. (2002). Attachment disturbances in young children I: The continuum of caretaking causality. Journal of the American Academy of Child and Adolescent Psychiatry, 41, 972-982.

UNICEF. Situação Mundial da Infância 2011: Caderno Brasil. Brasília, DF, 2011.

Wathier, J. L. \& Dell'Aglio, D. D. (2007). Sintomas depressivos e eventos estressores em crianças e adolescentes no contexto de institucionalização. Revista de Psiquiatria do Rio Grande do Sul, 29(3).

Wathier, J. L., Dell'Aglio, D. D., \& Bandeira, D. R. (2008). Análise fatorial do Inventário de Depressão Infantil (CDI) em amostra de jovens brasileiros. Avaliação Psicológica, 7(1), 75-84. Recuperado de <http://pepsic.bvsalud.org/scielo.php? script $=$ sci arttext\&pid $=$ S1677-04712008000100010\&lng $=$ pt $\&$ nrm $=$ iso $>$.

Wathier, J. L. \& Dell'Aglio, D. D. (2007). Sintomas depressivos e eventos estressores em crianças e adolescentes no contexto de institucionalização. Revista de Psiquiatria do Rio Grande do Sul, 29(3), 305-314. Recuperado de <http://www.scielo.br/scielo. php?script $=$ sci arttext\&pid $=$ S0101-81082007000300010\&lng $=$ en\&tlng $=$ pt. $10.1590 /$ S0101-81082007000300010 $>$.

Yunes, M. (2003). Psicologia positiva e resiliencia: o foco no indivíduo e na família. Psicologia em Estudo, 8, 1-15.

Zeanah, C. H., Smyke, A. T., Koga S. F., \& Carlson, E. (2005). Attachment in institutionalized and community children in Romania. Child Development, 76(5), 1015-1028. doi: 10.1111/ j.1467-8624.2005.00894.x

Autores:

Jordana Calcing - Mestre, Instituto de Desenvolvimento Educacional do Alto Uruguai.

Silvia Pereira da Cruz Benetti - Doutora, Universidade do Vale do Rio dos Sinos.

Endereço para correspondência:

Silvia Pereira da Cruz Benetti

Universidade do Vale do Rio dos Sinos

Av. Unisinos, 950

São Leopoldo, RS, Brasil

Recebido em: 29.04 .13

Aceito em: 30.07 .14 